\title{
Ice friction: Glacier sliding on hard randomly rough bed surface
}

Cite as: J. Chem. Phys. 149, 234701 (2018); https://doi.org/10.1063/1.5055934

Submitted: 12 September 2018 . Accepted: 09 November 2018 . Published Online: 18 December 2018

B. N. J. Persson

\section{COLLECTIONS}

This paper was selected as Featured
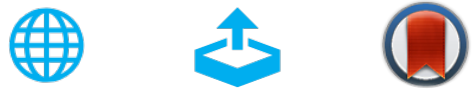

\section{ARTICLES YOU MAY BE INTERESTED IN}

Perspective: Searching for simplicity rather than universality in glass-forming liquids The Journal of Chemical Physics 149, 230901 (2018); https://doi.org/10.1063/1.5048093

Perspective: Excess-entropy scaling

The Journal of Chemical Physics 149, 210901 (2018); https://doi.org/10.1063/1.5055064

Common microscopic structural origin for water's thermodynamic and dynamic anomalies The Journal of Chemical Physics 149, 224502 (2018); https://doi.org/10.1063/1.5055908

PHYSICS TODAY WHITEPAPERS
ADVANCED LIGHT CURE ADHESIVES

Take a closer look at what these environmentally friendly adhesive systems can do
READ NOW

PRESENTED BY Q MASTERBOND: 


\title{
Ice friction: Glacier sliding on hard randomly rough bed surface
}

\author{
B. N. J. Persson \\ PGI-1, FZ Jülich, Jülich, Germany and Multiscale Consulting, Wolfshovener Str. 2, 52428 Jülich, Germany
}

(Received 12 September 2018; accepted 9 November 2018; published online 18 December 2018)

\begin{abstract}
I present a theory for ice friction for ice sliding on a hard randomly rough surface which includes ice melting-freezing (regelation), viscoelastic energy dissipation, and cavitation. The theory is an extension of earlier work by Weertman, Lliboutry, Nye, and Kamb. I present numerical results for surfaces with realistic surface roughness power spectra. I consider both airfilled and (pressurized) waterfilled cavities. The calculated frictional shear stresses are consistent with experimental observations for temperate glaciers. Published by AIP Publishing. https://doi.org/10.1063/1.5055934
\end{abstract}

\section{INTRODUCTION}

Mass loss from glaciers represents the largest (65\%) contribution to observed sea level rise. Fast glacier flow is mainly accomplished by localized motion at the ice-solid interface (called basal motion), involving complex sliding and shearing processes usually in the presence of water under high pressure and varying thermal conditions. However, due to the virtual inaccessibility of the subglacial environment, the sliding mechanisms are poorly understood.

Glacial dynamics are not completely accounted for in recent sea level projections. The core of the problem is to understand the complex spatio-temporal patterns and interactions at the interface between the moving glacier ice and the underlying bedrock. The response of the interface dynamics to external forcing, such as from surface melt water, is highly nonlinear as it strongly depends on the mechanical and thermal interface conditions, which in turn have a memory of earlier states. ${ }^{1,2}$

The motion of many glaciers and ice streams is controlled by frictional properties at the bed, which determine whether and how easily basal sliding will occur. Reliable sealevel-rise projections require an improved understanding of ice flow, in particular the slip at the ice-bed interface, which is currently one of the least understood aspects of glacier dynamics. Processes taking place at the ice-bed interface are poorly constrained by observation. In situ field measurements at the bed, from boreholes and tunnels, are very limited in spatial and temporal extent, while bed conditions are expected to vary considerably in time and space. Variations in the sliding style, including smooth sliding, stick-slip behavior, slowdowns, and switches of the flow state, are attributed to basal processes but are not well understood. A clearer understanding of the controls on the sliding rate, including time, temperature, and other basal characteristics, is essential to modeling and forecasting.

Temperature at the base of the glacier is controlled by heat conduction in response to geothermal heat flux, strain heating within the ice, and climate history and has a clear role in determining the mode and rate of glacier deformation. Warm-based (temperate) glaciers, those that are at their melting point at the ice-bed interface, are thought to deform by a combination of viscous flow and basal sliding. The sliding may be tied to the geometry and flow conditions of a basal hydrological system. The upper and central parts of a glacier flow more quickly than the bottom portions, where the friction of the valley walls and floor restrict its velocity from increasing. Cold-based glaciers, below freezing at the interface, are thought to be frozen to the bed, deforming through viscous flow in the bulk of the glacier only, rather than through basal sliding.

The friction between the glacier ice and the underlying bedrock has been studied in pioneering work by Weertman, ${ }^{3}$ Lliboutry, ${ }^{4}$ Nye,,${ }^{5,6}$ and Kamb. ${ }^{7}$ Some of these (and later) studies have assumed very idealized conditions such as cubic-like bedrock asperities. ${ }^{8}$ However, in two beautiful and very detailed studies, $\mathrm{Nye}^{5}$ and $\mathrm{Kamb}^{7}$ considered a more realistic model. Since the work of Nye and Kamb, contact mechanics and ice friction has developed a lot, and in this study, I will make use of a recently developed contact mechanics and rubber friction theory ${ }^{9-11}$ to study some aspects of ice friction in the context of the motion of glaciers. I consider the sliding of a thick ice slab (glacier) on a rigid substrate (bedrock) with a randomly rough surface with self-affine fractal properties. The model includes viscoelastic energy dissipation in the ice, regelation, and cavitation and predicts frictional shear stresses which are consistent with experimental observations.

\section{SOME BASIC FACTS ABOUT GLACIER DYNAMICS AND ICE RHEOLOGY}

The glaciers in Antarctica are typically $h \approx 1000 \mathrm{~m}$ thick and typically move (flow) with the speed of $\sim 10-100 \mathrm{~m} /$ year or $v \approx 3 \times 10^{-7}-3 \times 10^{-6} \mathrm{~m} / \mathrm{s}$. For a glacier in steady sliding (no acceleration), the frictional shear stress acting on the bottom surface of the ice (and on the bedrock) must equal $\tau_{\mathrm{f}}=\rho g h \sin \alpha$, where $\alpha$ is the slip angle (see Fig. 1). In a typical case, $\sin \alpha \approx 0.01$ giving $\tau_{\mathrm{f}} \approx 0.1 \mathrm{MPa}$. The normal pressure at the ice-rock interface we denote as the squeezing pressure is given by $p_{0} \approx \rho g h \cos \alpha$. For $h \approx 1000 \mathrm{~m}$, this gives $p_{0} \approx 10 \mathrm{MPa}$. 


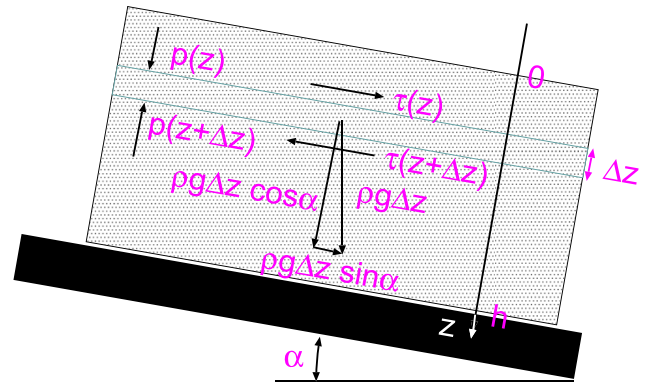

FIG. 1. Ice block on a tilted substrate. If the layer of thickness $\Delta z$ does not accelerate, force balance requires that the pressure $p(z)=\rho g z \cos \alpha$ and the tangential stress $\tau(z)=\rho g z \sin \alpha$. In particular, at the ice-rock interface, $p=p_{0}=\rho g h \cos \alpha$ and $\tau=\tau_{\mathrm{f}}=\rho g h \sin \alpha$.

The temperature at the bottom of the glaciers is often at the melting point of the ice. The physical reason for this is the low thermal conductivity of ice and the large thickness of the ice layer. The geothermal heat flux at the earth surface is about $J_{z} \approx 0.1 \mathrm{~J} \mathrm{~m}^{-2} \mathrm{~s}^{-1}$. If we assume that the temperature at the ice-rock interface is $T_{1}$ and at the upper ice-atmosphere surface is $T_{0}$, and if a steady state temperature profile prevails, then the heat current $J_{z}=\kappa\left(T_{1}-T_{0}\right) / h$, where $\kappa \approx 2 \mathrm{~J} \mathrm{~m}^{-1} \mathrm{~s}^{-1} \mathrm{~K}^{-1}$ is the thermal conductivity of ice. Using $J_{z}=0.1 \mathrm{~J} \mathrm{~m}^{-2} \mathrm{~s}^{-1}$, this gives a temperature difference of $T_{1}-T_{0} \approx 50{ }^{\circ} \mathrm{C}$. Thus, since the ice surface temperature (say averaged over a year) is above $T_{0} \approx-50{ }^{\circ} \mathrm{C}$, we predict that the ice will melt at the ice-rock interface, and the temperature will be equal to the ice melting temperature. In the steady state, the melt water will flow in channels at the icerock interface and remove thermal energy at such a rate that the total energy is conserved. In addition to the geothermal energy flux, frictional energy dissipation will occur at the sliding ice-rock interface and contribute to a heat current of similar magnitude as the geothermal one (the frictional heat current $\tau_{\mathrm{f}} \approx 0.03-0.3 \mathrm{~J} \mathrm{~m}^{-2} \mathrm{~s}^{-1}$ ).

For perfectly smooth surfaces, when the ice temperature is at the ice melting temperature, one expects a extremely small frictional shear stress since even a nanometer of water film between the ice and the countersurface results in a frictional shear stress $\tau_{\mathrm{f}}=\eta v / d \approx 1 \mathrm{~Pa}$, which is a factor of $\sim 10^{5}$ times smaller than typically observed for glaciers. ${ }^{12}$ Here we have used the water viscosity $\eta \approx 0.001$ Pas and sliding speed $v \approx 10^{-6} \mathrm{~m} / \mathrm{s}$. The actual shear stress is determined by the surface roughness of the rockbed and will be discussed below.

When ice is deformed rapidly with small strain, it responds as an elastically stiff solid with Young's modulus $E$ of order several GPa. When the strain reaches a large enough value, ice deforms plastically. ${ }^{13}$ The strain and stress at the onset of plastic flow depend on the deformation speed or strain rate. For a small strain rate, experiments have shown that to a good approximation, the stress-strain rate relation (Glen's law, see Ref. 14) is

$$
\dot{\epsilon}_{i j}=A \tau^{n-1} \tau_{i j}
$$

where $\epsilon_{i j}$ and $\tau_{i j}$ are the deviatoric strain and stress, respectively, and $2 \tau^{2}=\tau_{i j} \tau_{i j}$ (summation over repeated indices is implicitly understood). At the melting point of ice,
$A \approx 2.4 \times 10^{-24} \mathrm{~Pa}^{-3} \mathrm{~s}^{-1}$ and $n \approx 3$. We can also write (1) as

$$
\tau_{i j}=2 \eta \dot{\epsilon}_{i j}
$$

where the (effective) viscosity

$$
\eta=\frac{1}{2 A \tau^{n-1}}
$$

or with $\dot{\epsilon}^{2}=\dot{\epsilon}_{i j} \dot{\epsilon}_{i j}$

$$
\eta=2^{-(n+1) /(2 n)} A^{-1 / n} \dot{\epsilon}^{(1-n) / n}
$$

depends on the strain rate as for a non-Newtonian (shearthinning) fluid. If the strain rate in an application takes some particular value, one may approximately treat the viscosity as strain-rate independent, as obtained from (2) for the relevant strain rate. This approach is often used in applications to glacier friction.

\section{THEORY}

Consider a viscoelastic solid (ice) with a flat surface squeezed against a rigid solid (rock) with a randomly rough surface. We assume a small slope approximation, i.e., the rootmean-square slope of the rough surface is much smaller than unity. Assume that the solids move with the relative lateral speed $\mathbf{v}$, and consider that the system is in a reference frame where the lower solid moves with the velocity $\mathbf{v}$, while the center of mass of the viscoelastic solid is stationary. The ice at the interface with the rock will melt due to pressure melting. If we assume that the melt water is able to get removed (squeezed-out) from the ice-rock contact regions, then the normal velocity at the ice-rock interface must equal the melt front velocity which we denote with $\dot{w}(\mathbf{x}, t)$ (see Fig. 2). Thus, the bottom surface of the upper solid (ice) has the normal displacement

$$
u_{z}(\mathbf{x}, t)=h(\mathbf{x}-\mathbf{v} t)+w(\mathbf{x}, t) .
$$

If we define the Fourier transform

$$
f(\mathbf{q}, \omega)=\frac{1}{(2 \pi)^{3}} \int d^{2} x d t f(\mathbf{x}, t) e^{-i(\mathbf{q} \cdot \mathbf{x}-\omega t)},
$$

we get

$$
u_{z}(\mathbf{q}, \omega)=h(\mathbf{q}, \omega)+w(\mathbf{q}, \omega),
$$

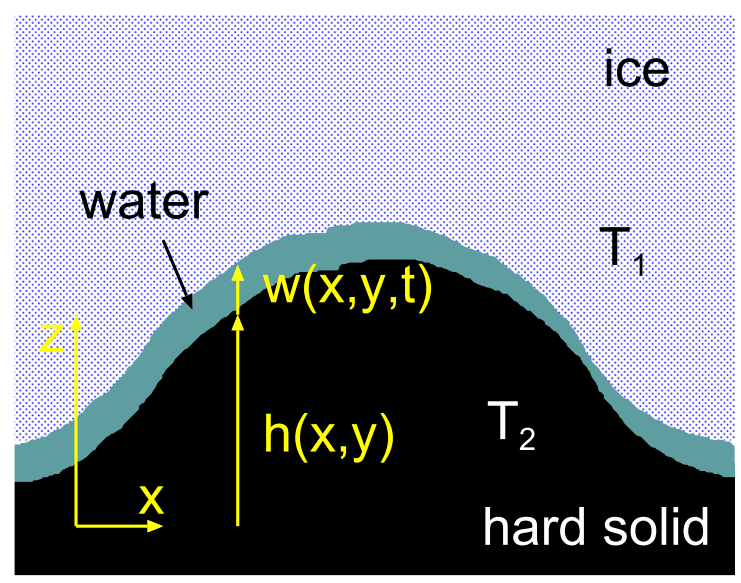

FIG. 2. An ice block (semi-infinite solid) sliding on a rigid solid with a randomly rough surface. A thin water film separates the solids. 
where $h(\mathbf{q}, \omega)=h(\mathbf{q}) \delta(\omega-\mathbf{q} \cdot \mathbf{v})$. If we assume the stress $\sigma_{z z}(\mathbf{q}, \omega)$ act on the bottom surface of the ice, then

$$
M_{z z}(\mathbf{q}, \omega) \sigma_{z z}(\mathbf{q}, \omega)=u_{z}(\mathbf{q}, \omega),
$$

where for a linear viscoelastic solid at low frequencies, ${ }^{9}$

$$
M_{z z}(\mathbf{q}, \omega)=-\frac{2\left(1-v^{2}\right)}{E(\omega) q}
$$

Combining (3) and (4) gives

$$
M_{z z}(\mathbf{q}, \omega) \sigma_{z z}(\mathbf{q}, \omega)=h(\mathbf{q}, \omega)+w(\mathbf{q}, \omega) .
$$

We assume that the thickness of the boundary layer between the two solids increases due to pressure melting and decreases due to refreezing, but we treat it as an external film in what follows. Let $T_{1}(\mathbf{x}, z, t)$ refer to the temperature in the ice and $T_{2}(\mathbf{x}, z, t)$ in the rock. When the film makes a transition from fluid to solid, the heat per unit surface area and unit time given off is $L \dot{w}$, where $L$ is the latent heat per unit volume of ice. Similarly when the ice melts, the energy $L \dot{w}$ is adsorbed per unit surface area and unit time. This energy acts as a (positive or negative) heat source $\dot{Q}(\mathbf{x}, t)=L \dot{w}$ at the interface between the two solids. The heat flow in the solids satisfies

$$
\frac{\partial T}{\partial t}-D \nabla^{2} T=0
$$

We assume that the boundary film is very thin so that the temperature at the interface between the ice and the rock is continuous $\left(T_{1}=T_{2}\right)$. Energy conservation gives the second boundary condition $\kappa_{1} d T_{1} / d z-\kappa_{2} d T_{2} / d z=\dot{Q}$. Using the equations above, we get the temperature in the boundary film,

$$
T(\mathbf{q}, \omega)=\frac{-L(-i \omega)}{\kappa_{1} \gamma_{1}+\kappa_{2} \gamma_{2}} w(\mathbf{q}, \omega),
$$

where

$$
\gamma_{1}=\left(q^{2}-i \omega / D_{1}\right)^{1 / 2}
$$

and similar for $\gamma_{2}$. Here $D_{1}=\kappa_{1} / \rho_{1} C_{V 1}$, where $\kappa_{1}, \rho_{1}$, and $C_{V 1}$ are the thermal conductivity, mass density, and heat capacity of the ice respectively. In a similar way, the heat diffusivity $D_{2}$ of the rock is defined.

If the temperature $T$ is measured relative to the (average) melting point temperature, we can write the temperature in the boundary film as $T=-S p$, where $p=\sigma_{z z}$ is the pressure (relative to the average pressure) at the bottom surface of the ice. Thus, we get

$$
N(\mathbf{q}, \omega) \sigma_{z z}(\mathbf{q}, \omega)=-w(\mathbf{q}, \omega),
$$

where

$$
N(\mathbf{q}, \omega)=\frac{S\left(\kappa_{1} \gamma_{1}+\kappa_{2} \gamma_{2}\right)}{i \omega L} .
$$

Using (6) and (7) gives

$$
\bar{M}_{z z}(\mathbf{q}, \omega) \sigma_{z z}(\mathbf{q}, \omega)=h(\mathbf{q}, \omega)
$$

where

$$
\bar{M}_{z z}(\mathbf{q}, \omega)=M_{z z}(\mathbf{q}, \omega)+N(\mathbf{q}, \omega)
$$

Thus, the only role of the (water) boundary film on the contact mechanics is to renormalize the coupling between the normal stress and the displacement at the ice-rock interface. Using (5), (8), and (10), we can write

$$
\bar{M}_{z z}(\mathbf{q}, \omega)=-\frac{2\left(1-v^{2}\right)}{E(\omega) q}\left[1+\frac{S\left(\kappa_{1} \gamma_{1}+\kappa_{2} \gamma_{2}\right) E(\omega) q}{(-i \omega) 2\left(1-v^{2}\right) L}\right] .
$$

We assume a Maxwell model for the rheological properties of ice. ${ }^{15,16}$ Thus, the shear modulus is (see Fig. 3)

$$
\frac{1}{G(\omega)}=\frac{1}{G_{0}}+\frac{1}{(-i \omega) \eta}
$$

where the low-strain and high-frequency shear modulus is $G_{0}$ $\approx 1 \mathrm{GPa}$ and the (effective) viscosity at the melting point of ice is $\eta \approx 3 \times 10^{13} \mathrm{Pas}$. In earlier studies of glacier sliding friction, the elastic response of the ice (the $G_{0}$-term) was neglected. Glaciers usually slide at very low velocities, but, at least in the absence of regelation, the bedrock roughness with a large wavenumber $q$ (or short wavelength) gives rise to high perturbing frequencies, $\omega \approx q v$, where the ice respondence could be elastic rather than viscous. Including the $G_{0}$-term in $G(\omega)$ will indicate at which slip velocities, the elastic response may matter (see Sec. IV).

Using $E=2(1+v) G$, we get

$$
\frac{1}{E(\omega)}=\frac{1}{E_{0}}+\frac{1}{(-i \omega) 2(1+v) \eta} .
$$

If we consider very low frequencies so that $E_{0} \gg \omega \eta$ and $q^{2} \gg \omega / D$ (so that $\gamma_{1} \approx \gamma_{2} \approx q$ ), we get from (11) and (12)

$$
\bar{M}_{z z}(\mathbf{q}, \omega)=\frac{\left(1-v^{2}\right)}{i \omega(1+v) \eta q}\left[1+\left(\frac{q}{q_{*}}\right)^{2}\right],
$$

where

$$
q_{*}^{2}=\frac{2\left(1-v^{2}\right) L}{S\left(\kappa_{1}+\kappa_{2}\right) 2(1+v) \eta} .
$$

Defining the average thermal conductivity $\kappa=\left(\kappa_{1}+\kappa_{2}\right) / 2$ and assuming incompressible system $(v=0.5)$, we get

$$
\begin{gathered}
\bar{M}_{z z}(\mathbf{q}, \omega)=\frac{1}{2 i \omega \eta q}\left[1+\left(\frac{q}{q_{*}}\right)^{2}\right], \\
q_{*}^{2}=\frac{L}{4 S \kappa \eta} .
\end{gathered}
$$

In Ref. 9, I have shown that for the case the surface displacement of a viscoelastic solid is related to the surface stress with a relation of the form (4), the frictional shear stress is

$$
\tau_{\mathrm{f}}=\int d^{2} q q_{x} C(\mathbf{q}) P(q) S(q) \operatorname{Im}\left[\bar{M}_{z z}\left(\mathbf{q}, q_{x} v\right)\right]^{-1},
$$

where $^{17,18}$

$$
S(q)=\gamma+(1-\gamma) P^{2}(q)
$$

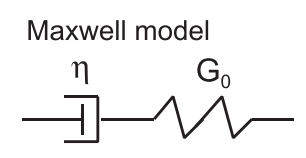

FIG. 3. Simple rheology model of ice. 
where $\gamma \approx 0.4$ and

$$
P(q)=\operatorname{erf}\left(\frac{1}{2} G^{-1 / 2}(q)\right)
$$

with

$$
G\left(q^{\prime}\right)=\frac{1}{2} \int_{q_{0}<q<q^{\prime}} d^{2} q C(\mathbf{q})\left|p_{0} \bar{M}_{z z}\left(\mathbf{q}, q_{x} v\right)\right|^{-2},
$$

where $p_{0}$ is the nominal squeezing pressure at the interface between the two solids (here ice and bedrock). The surface roughness power spectrum is ${ }^{19-21}$

$$
C(\mathbf{q})=\frac{1}{(2 \pi)^{2}} \int d^{2} x\langle h(\mathbf{x}) h(\mathbf{0})\rangle e^{-i \mathbf{q} \cdot \mathbf{x}},
$$

where $\langle\ldots\rangle$ stands for ensemble averaging. We have assumed $\langle h(\mathbf{x})\rangle=0$. For surfaces with roughness with isotropic statistical properties, the power spectrum depends only on the magnitude $q=|\mathbf{q}|$ of the wavevector. The integral in (15) is for $q_{0}<q<q_{1}$, where the small and large wavenumber cutoffs, $q_{0}$ and $q_{1}$, depend on the system under consideration. Thus, in the present case, $q_{0}$ is determined by the thickness of the ice layer, $q_{0}=2 \pi / h$. The large wavenumber cutoff could be $q_{1}=2 \pi / d$, where $d$ is an atomic distance, or the length scale where the continuum description of the mechanical properties of the solids break down. However, in the present case, there is an effective cutoff determined by the regelation process which results in $d$ of order a few mm (see Sec. IV). The integral (18) is between $q_{0}<q<q^{\prime}$.

Sometimes the surface topography is measured along a straight line rather than over a square surface area. In this case, one can calculate the one-dimensional (1D) surface roughness power spectrum

$$
C_{1 \mathrm{D}}=\frac{1}{2 \pi} \int d x\langle h(x) h(0)\rangle e^{-i q x} .
$$

For surfaces with roughness having isotropic statistical properties, it is easy to obtain the $2 \mathrm{D}$ power spectrum $C(q)$ from the $1 \mathrm{D}$ power spectrum. ${ }^{10,19}$

Assuming $p_{0}$ so large that complete contact occurs (no cavitation) $P=S=1$, we get from (13) and (15)

$$
\tau_{\mathrm{f}}=\int d^{2} q C(q) \frac{2 \eta v q_{x}^{2} q}{1+\left(q / q_{*}\right)^{2}} .
$$

This limiting case agrees with the result derived by $\mathrm{Nye}^{5}$ and Kamb. ${ }^{7}$

\section{NUMERICAL RESULTS}

When sliding at high enough sliding speed, surface separation occurs in some fraction of the nominal contact region. The non-contact regions are usually referred to as cavities. Depending on the situation, the cavities can be filled with air (but rather unlikely situation), or with pressurized water. In the latter case, some part of the weight of the ice glacier will be carried by the pressurized water. We consider these two situations separately.
We assume that the ice can be described using the Maxwell rheological model where the viscoelastic modulus is given by (12). We choose the viscosity $\eta=3 \times 10^{13} \mathrm{Pas}$ and $E_{0}=3 \mathrm{GPa}$ or $E_{0}=\infty$. We also need information about the bedrock surface roughness. We assume that the bedrock is randomly rough in which case the surface roughness power spectrum contains all the statistical information about the surface topography.

\section{A. Bedrock surface roughness power spectrum}

Figure 4 shows the one-dimensional (1D) power spectrum as a function of the wavenumber (log-log scale) of a glacier bedrock surface obtained from measurements ${ }^{22}$ of the surface topography at two different locations (a) and (b). The solid lines are along the glacier sliding direction, and the dots are orthogonal to the sliding direction. The dashed line corresponds to a self-affine fractal surface with the Hurst exponent $H=2$ (fractal dimension $D_{\mathrm{f}}=2$ ), and the dotted line corresponds to $H=0.8$ (or $D_{\mathrm{f}}=2.2$ ). The Hurst exponent $\approx 0.8$ is typical for many surfaces such as surfaces produced by fracture or grinding. The root-mean-square roughness is about $0.1 \mathrm{~m}$.

In the following calculations, we have used the $2 \mathrm{D}$ power spectra shown in Fig. 5. This power spectra correspond to surfaces with self-affine fractal properties with the rootmean-square (rms) roughness $0.1 \mathrm{~m}$ and the Hurst exponent
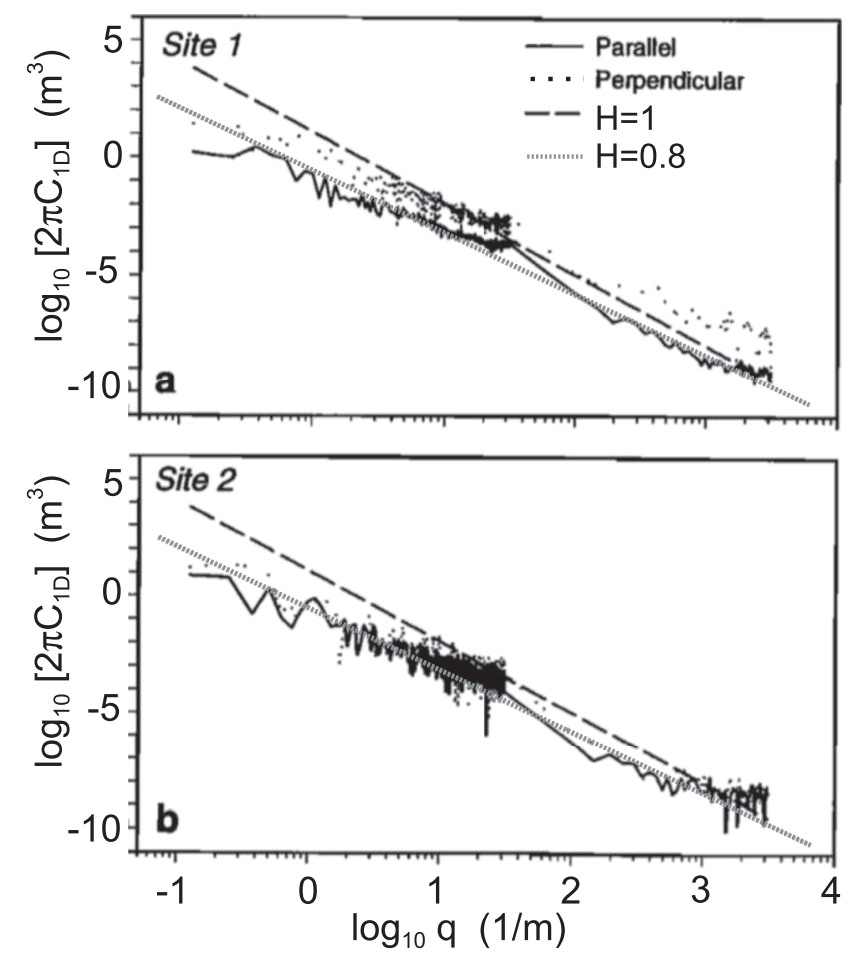

FIG. 4. The one-dimensional (1D) surface roughness power spectrum $C_{1 \mathrm{D}}(q)$ as a function of the wavenumber (log-log scale) of a glacier bedrock surface obtained from measurements of the surface topography at two different locations (a) and (b). The solid lines are along the glacier sliding direction, and the dots are orthogonal to the sliding direction. The dashed line corresponds to a self-affine fractal surface with the Hurst exponent $H=2$ (fractal dimension $D_{\mathrm{f}}=2$ ), and the dotted line corresponds to $H=0.8\left(\right.$ or $D_{\mathrm{f}}=2.2$ ). The Hurst exponent $\approx 0.8$ is typical for many surfaces such as surfaces produced by fracture or grinding. Adapted from Ref. 22. 


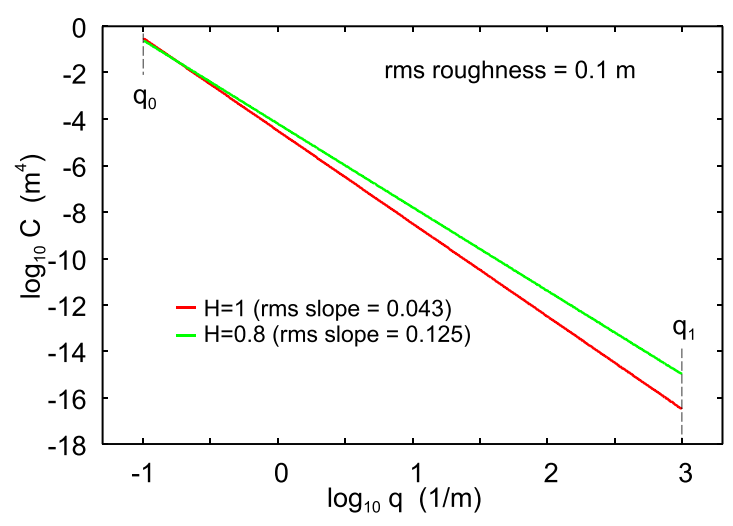

FIG. 5. The 2D surface roughness power spectra as a function of the wavenumber (log-log scale) for a surface with self-affine fractal properties with the Hurst exponent $H=1$ (red line) and $H=0.8$ (green line). The power spectra correspond to the surface root-mean-square (rms) roughness $0.1 \mathrm{~m}$ and the rms slope 0.043 and 0.125 , respectively. The smallest and largest wavenumbers are denoted $q_{0}$ and $q_{1}$, respectively. In the present case, $q_{0}=0.1 \mathrm{~m}^{-1}$ and $q_{1}=10^{3} \mathrm{~m}^{-1}$, corresponding to the wavelength $\lambda_{0}=2 \pi / q_{0} \approx 63 \mathrm{~m}$ and $\lambda_{1} \approx 0.63 \mathrm{~cm}$, respectively.

$H=1$ (red line) and $H=0.8$ (green line). The rms slopes of the corresponding surfaces are 0.043 and 0.125 , respectively. The $1 \mathrm{D}$ power spectra for these surfaces are similar to the power spectra in Fig. 4.

\section{B. Airfilled cavities}

We first present numerical results for the frictional shear stress as a function of the sliding speed at the glacier bedrock interface, assuming air-filled cavities. In Fig. 6, we show the (nominal) frictional shear stress (a) and the relative contact area (b) as a function of the logarithm of the sliding speed. We have used the latent heat $L=3 \times 10^{8} \mathrm{~J} \mathrm{~m}^{-3}$, the mean thermal conductivity $\kappa=2 \mathrm{~J} \mathrm{~m}^{-1} \mathrm{~K}^{-1} \mathrm{~s}^{-1}$, and $S=7 \times 10^{-8} \mathrm{KPa}^{-1}$, and the Maxwell rheological model for the ice with $\eta=10^{13}$ Pas and $E_{0}=3 \mathrm{GPa}$ (dashed lines) and $E_{0}=\infty$ (solid lines). We have assumed that the nominal contact pressure $p_{0}=10$ MPa acts at the bottom surface of the ice, corresponding to the weight of an $\approx 1000 \mathrm{~m}$ thick ice layer.

Figure 7 shows the (nominal) frictional shear stress as a function of the logarithm of the upper cut-off wavenumber $q_{1}$ for the sliding speed $v=10^{-6} \mathrm{~m} / \mathrm{s}$. We show results for the two power spectra shown in Fig. 5 corresponding to $H=1$ (red line) and $H=0.8$ (green line). We have used the Maxwell rheological model for the ice with $\eta=10^{13}$ Pas and $E_{0}=\infty$, but as shown in Fig. 6, the same result prevails for $E_{0}=3 \mathrm{GPa}$ at this sliding speed.

Note that for $H=1$, including roughness at larger wavenumbers than $q_{1}=10^{3} \mathrm{~m}^{-1}$ does not increase the frictional shear stress. This is due to the regelation which effectively removes the viscoelastic contribution to the friction from the short wavelength roughness. Thus, instead of deforming viscoelastically in response to the short wavelength roughness, the ice locally melts and refreezes, which effectively eliminates the viscoelastic deformations at a short enough length scale. The same is true for $H=0.8$, but here the effective cutoff is shifted to a slightly larger wavenumber. This effect is caused by the fact that decreasing the Hurst exponent (but keeping the rms-roughness amplitude fixed) results in surfaces
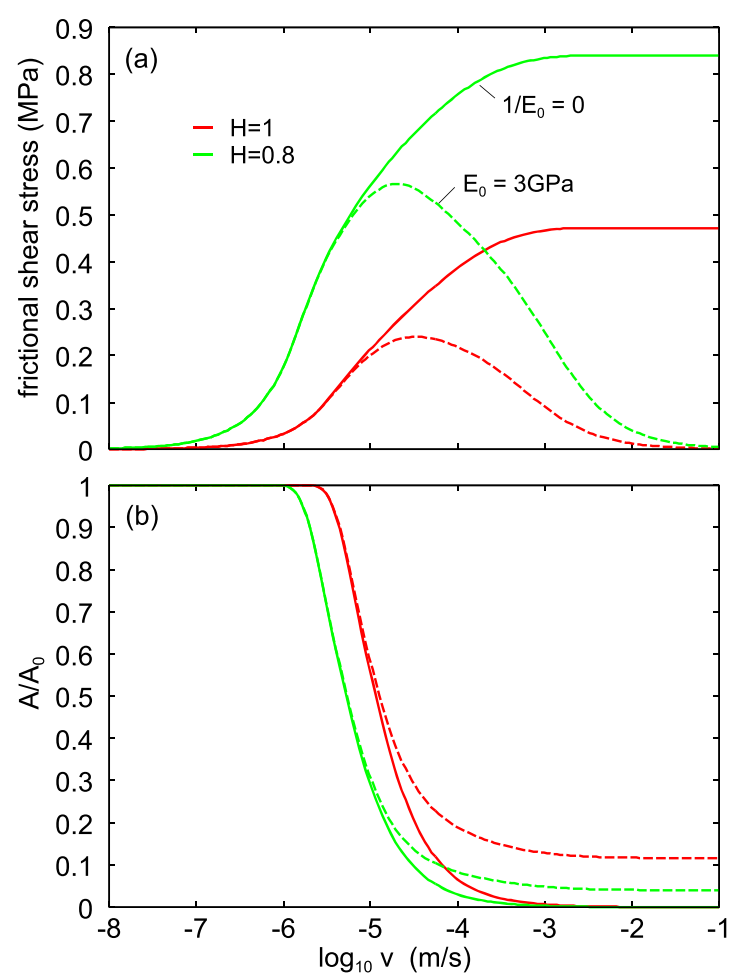

FIG. 6. The (nominal) frictional shear stress (a) and the relative contact area (b) as a function of the logarithm of the sliding speed. We have used the latent heat $L=3 \times 10^{8} \mathrm{~J} \mathrm{~m}^{-3}$, the mean thermal conductivity $\kappa=2 \mathrm{~J} \mathrm{~m}^{-1} \mathrm{~K}^{-1} \mathrm{~s}^{-1}$, and $S=7 \times 10^{-8} \mathrm{KPa}^{-1}$. We have used the Maxwell rheological model for the ice with $\eta=10^{13}$ Pas and $E_{0}=3 \mathrm{GPa}$ (dashed lines) and $E_{0}=\infty$ (solid lines).

with an increasing amplitude of the large wavenumber (or short wavelength) roughness, which are harder to remove by regelation.

\section{Waterfilled cavities}

We assume that the non-contact regions (cavities) are filled with pressurized water. We assume that the water pressure $p_{\mathrm{w}}$ is the same everywhere in all the cavities at the ice-rock interface. We take into account the water pressure in a mean

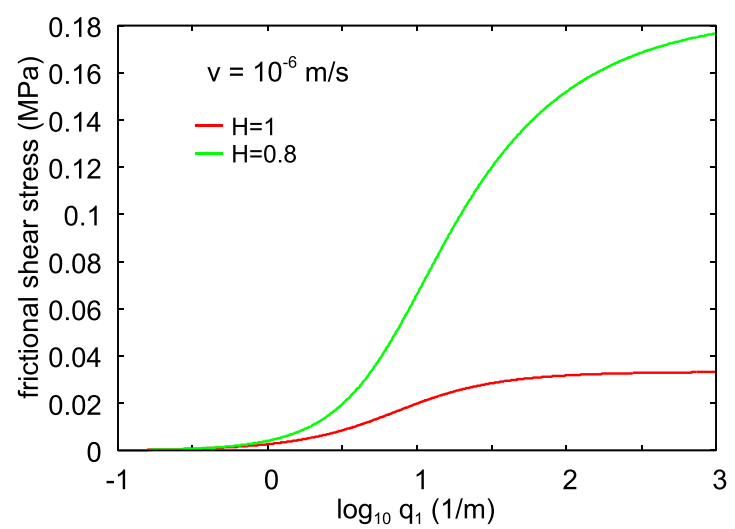

FIG. 7. The (nominal) frictional shear stress as a function of the logarithm of the upper cut-off wavenumber $q_{1}$ for the sliding speed $v=10^{-6} \mathrm{~m} / \mathrm{s}$. We show results for the two power spectra shown in Fig. 5 corresponding to $H=1$ (red line) and $H=0.8$ (green line). We have used the latent heat $L=3$ $\times 10^{8} \mathrm{~J} \mathrm{~m}^{-3}$, the mean thermal conductivity $\kappa=2 \mathrm{~J} \mathrm{~m}^{-1} \mathrm{~K}^{-1} \mathrm{~s}^{-1}$, and $S=7$ $\times 10^{-8} \mathrm{KPa}^{-1}$. We have used the Maxwell rheological model for the ice with $\eta=10^{13}$ Pas and $E_{0}=\infty$. 
field manner by assuming that the squeezing pressure is given by $p_{0}-p_{w}\left(A_{0}-A\right) / A_{0}$, i.e., we reduce the load $p_{0} A_{0}$ given by the weight of the ice with the upward acing fluid pressure force $p_{\mathrm{w}}\left(A_{0}-A\right)$. Here $A$ is the (projected) ice-rock contact area and $A_{0}$ is the nominal contact area, i.e., the surface area of the bottom surface of the ice projected on the $x y$-plane.

Figure 8 shows the (nominal) frictional shear stress (a) and the fraction of the total load carried by the water (b) as a function of the logarithm of the sliding speed. As expected, when the water pressure in the cavities increases, the frictional shear stress decreases and the fraction of the total load carried by the water increases. When the water pressure increases toward the nominal contact pressure $p_{0}$, caused by the weight of the ice, lift-off occurs where the ice is floating on a water layer and the frictional shear stress vanishes.

In the calculation above, we have assumed that the water pressure $p_{\mathrm{w}}$ is the same everywhere as expected if all the cavities are interconnected and the viscous pressure drop associated with fluid flow is negligible. If the water at the ice-rock interface is connected by water filled channels (e.g., cracks) to the upper surface of the ice block, then the water pressure at the ice-rock interface will be equal to the nominal pressure $p_{0}$ resulting from the weight of the ice (or slightly more because of the larger mass density of water than of ice). In this case, lift-off would occur where the friction would nearly vanish and where the ice block (glacier) could slip with "high" speed downhills. This type of rapid motion has been observed.
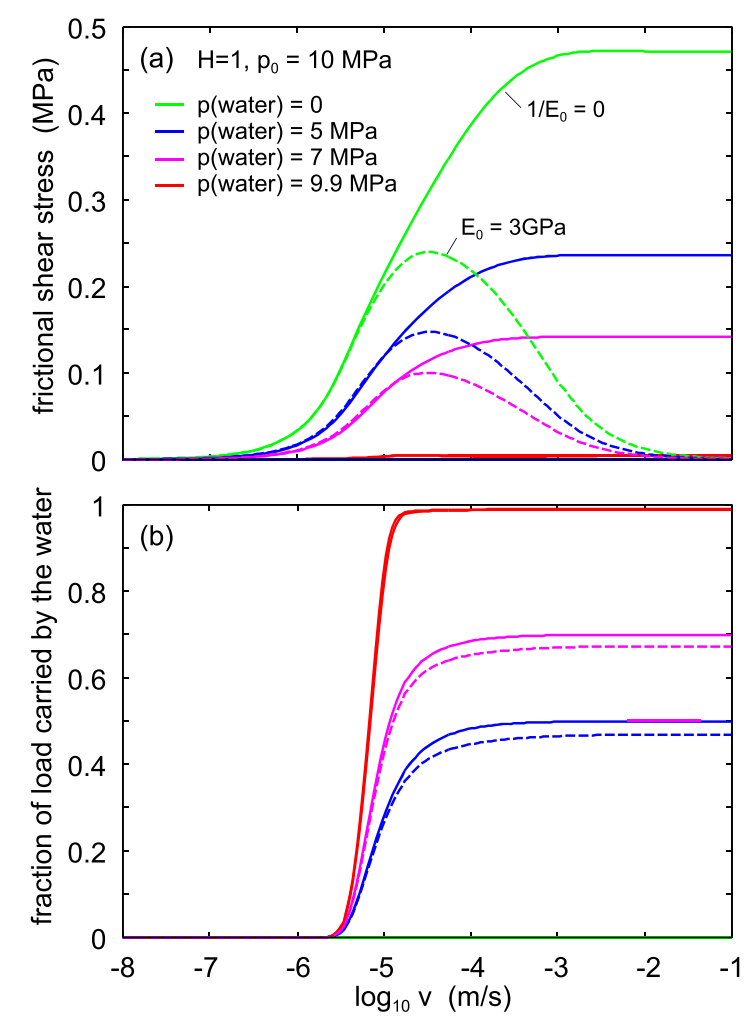

FIG. 8. The (nominal) frictional shear stress (a) and the fraction of the total load carried by the water (b) as a function of the logarithm of the sliding speed. We have used the latent heat $L=3 \times 10^{8} \mathrm{~J} \mathrm{~m}^{-3}$, the mean thermal conductivity $\kappa=2 \mathrm{~J} \mathrm{~m}^{-1} \mathrm{~K}^{-1} \mathrm{~s}^{-1}$, and $S=7 \times 10^{-8} \mathrm{KPa}^{-1}$. We have used the Maxwell rheological model for the ice with $\eta=10^{13} \mathrm{Pas}$ and $E_{0}=3 \mathrm{GPa}$ (dashed lines) and $E_{0}=\infty$ (solid lines).

\section{DISCUSSION}

In the model calculation above, we have used the Maxwell rheology model. In the model a small strain (and high strain rate) ice Young's modulus $E_{0}$ occurs. However, this description of ice rheology is only valid as long as the strain is very small, which is, in general, not the case in our applications. Thus, for large strain (and large strain rate), ice may fracture in a brittle manner, or undergo plastic deformation of a type which cannot be described by Glen's law (1). (In the present case, brittle fracture may be not important due to the large hydrostatic pressure at the ice-rock interface.) Nevertheless, including $E_{0}$ indicates the range of sliding speeds where the rheology may be properly described by the effective viscosity term alone in the Maxwell rheology expression for $E(\omega)$. Thus, Figs. 6 and 8 show that only for sliding speeds $v>10^{-5} \mathrm{~m} / \mathrm{s}$ does the $E_{0}$ term become important, and these high sliding speeds are usually not observed for the motion of glaciers.

The assumption made above that the water pressure at the ice-rock interface is constant everywhere is unlikely to be accurate in general. The water pressure depends on the size and distribution of flow channels at the interface. This is a very complex topic as the flow channels depend on the ice melting in response to frictional heating (and the geothermal heat current). Thus, the theories ${ }^{23}$ which have recently been developed for the leakage of seals cannot be directly applied to the interfacial fluid flow problem for glaciers. In particular, the observation that for randomly rough surfaces, the area of contact percolates [and the fluid flow (leakage) vanishes] when the relative contact area is $\approx 42 \%$ of the nominal contact area ${ }^{24}$ has probably no relevance for the flow of water at the ice-rock interface of glaciers.

The theory above includes cavitation. The multiscale contact mechanics theory used above has been tested in the past by comparing to exact numerical simulations, ${ }^{25}$ and the results never deviate with more than $\approx 20 \%$ from the simulation results. However, in the present case, there is an additional effect, namely, regelation, which was not included in the earlier studies and which may complicate the situation. More studies are needed to test the influence of regelation on the cavitation process.

One contribution to the glacier friction will be the friction between stone fragments which are embedded in the ice at the ice-bedrock interface. These stone fragments will slide with the ice and give a stone-stone sliding friction contribution to the glacier friction. In addition, it will polish or grind the bedrock surface in a similar way as the action of sandpaper. This wear process may determine the (short and medium wavelength) roughness of the bedrock.

In Ref. 6, Nye argued that the surface roughness power spectrum of the bedrock should scale with the wavenumber as $C(q) \sim q^{-4}$ (or $\sim q^{-3}$ for the 1D power spectrum). Since for a self-affine fractal surface, $C(q) \sim q^{-2(1+H)}$, this correspond to the Hurst exponent $H=1$ (and the fractal dimension $D_{\mathrm{f}}=3-H=2$ ). Such a surface has the scaling property that if some region is magnified, the roughness observed looks the same as the roughness at a larger length scale, i.e., the ratio between the amplitude and the wavelength does (on the average) not change. We now know this is, in general, not 
the case although some surfaces, e.g., sandblasted surfaces, typically have $H \approx 1$ (see Ref. 21 ). Surfaces prepared by fracture (crack propagation) or by grinding (or polishing) tend to have the Hurst exponent $H \approx 0.7-0.8$ (fractal dimension $D_{\mathrm{f}} \approx 2.2-2.3$ ). This appears to be the case also for the rockbed of glaciers (see Fig. 4). For such surfaces, the ratio between the amplitude and the wavelength of the surface roughness increases as the wavelength decreases.

It is interesting to consider testing the theory presented above in the laboratory. We have shown above that regelation removes the contribution to the friction from the surface roughness components with wavelengths smaller than $\approx 6 \mathrm{~cm}$ when the Hurst exponent is equal to $H=1$ and $\approx 6 \mathrm{~mm}$ when $H=0.8$. Clearly, ice sliding friction studies on sandblasted surfaces (which typically have $H \approx 1$ ) are not interesting as such surfaces have only short-wavelength roughness (with wavelength typically lower than $\approx 1 \mathrm{~mm}$ ). However, surfaces produced by fracture (crack propagation) of granite blocks will have roughness extending to the linear size of the block and typically with the Hurst exponent $H \approx 0.8$. If rectangular ice blocks with the linear dimension of order $10-30 \mathrm{~cm}$ are slid on such surfaces at the ice melting temperature, it should be possible to test the theory predictions at least in an approximate way.

\section{SUMMARY AND CONCLUSION}

I have presented a theory for ice friction for ice sliding on a hard randomly rough surface which includes ice meltingfreezing (regelation), viscoelastic energy dissipation, and cavitation. The theory is an extension of earlier work by Weertman, Lliboutry, Nye, and Kamb. I have presented numerical results for surfaces with realistic surface roughness power spectra. I have considered both airfilled and (pressurized) waterfilled cavities. The calculated frictional shear stresses are consistent with experimental observations for temperate glaciers.

\section{ACKNOWLEDGMENTS}

I thank Angelika Humbert (Alfred-Wegener-Institut für Polar- und Meeresforschung, Bremerhaven) for drawing my attention to the fascinating topic of glacier friction and for interesting discussions. I also thank J. F. Nye for useful comments on the manuscript and discussions.

${ }^{1}$ C. J. Van Der Veen, L. A. Stearns, J. Johnson, and B. Csatho, "Flow dynamics of byrd glacier, east Antarctica," J. Glaciol. 60, 1053 (2014).

${ }^{2} \mathrm{P}$. W. Thorp, "Surface profiles and basal shear stresses of outlet glaciers front a Late-glacial mountain ice field in western Scotland," J. Glaciol. 37, 77 (1991).
${ }^{3}$ J. Weertman, "On the sliding of glaciers," J. Glaciol. 3, 33 (1957).

${ }^{4}$ L. Lliboutry, "General theory of subglacial cavitation and sliding of temperate glaciers," J. Glaciol. 7, 21 (1968).

${ }^{5}$ J. F. Nye, "A calculation on the sliding of ice over a wavy surface using a Newtonian viscous approximation," Proc. R. Soc. A 311, 445 (1969).

${ }^{6}$ J. F. Nye, "Glacier sliding without cavitation in a linear viscous approximation," Proc. R. Soc. A 315, 381 (1970).

${ }^{7}$ B. Kamb, "Sliding motion of glaciers: Theory and observation," Rev. Geophys. Space Phys. 8, 673, https://doi.org/10.1029/rg008i004p00673 (1970)

${ }^{8}$ J. Weertman, "The unsolved general glacier sliding problem," J. Glaciol. 23, 97 (1979).

${ }^{9}$ B. N. J. Persson, "Theory of rubber friction and contact mechanics," J. Chem. Phys. 115, 3840 (2001).

${ }^{10}$ G. Carbone, B. Lorenz, B. N. J. Persson, and A. Wohlers, "Contact mechanics and rubber friction for randomly rough surfaces with anisotropic statistical properties," Eur. Phys. J. E 29, 275 (2009).

${ }^{11}$ B. N. J. Persson, "Contact mechanics for randomly rough surfaces," Surf. Sci. Rep. 61, 201 (2006).

${ }^{12}$ B. N. J. Persson, "Ice friction: Role of non-uniform frictional heating and ice premelting," J. Chem. Phys. 143, 224701 (2015).

${ }^{13}$ See, e.g., Sec. 14.2 in, B. N. J. Persson, Sliding Friction: Physical Principles and Applications (Springer, Heidelberg, 2000).

${ }^{14}$ J. W. Glen, "The creep of polycrystalline ice," Proc. R. Soc. London, Ser. A 228, 519 (1955).

${ }^{15} \mathrm{G}$. H. Gudmundsson, "Ice-stream response to ocean tides and the form of the basal sliding law," Cryosphere 5, 259 (2011).

${ }^{16}$ J. Christmann, C. Plate, R. Müller, and A. Humbert, "Viscous and viscoelastic stress states at the calving front of Antarctic ice shelves," Ann. Glaciol. 57, 10 (2016).

${ }^{17}$ M. Scaraggi and B. N. J. Persson, "Friction and universal contact area law for randomly rough viscoelastic contacts," J. Phys.: Condens. Matter 27, 105102 (2015).

${ }^{18}$ A. Almqvist, C. Campana, N. Prodanov, and B. N. J. Persson, "Interfacial separation between elastic solids with randomly rough surfaces: Comparison between theory and numerical techniques," J. Mech. Phys. Solids 59, 2355 (2011).

${ }^{19}$ P. R. Nayak, "Random process model of rough surfaces," J. Lubr. Technol. 93, 398 (1971).

${ }^{20}$ B. N. J. Persson, O. Albohr, U. Tartaglino, A. I. Volokitin, and E. Tosatti, "On the nature of surface roughness with application to contact mechanics, sealing, rubber friction and adhesion," J. Phys.: Condens. Matter 17, R1 (2005)

${ }^{21}$ B. N. J. Persson, "On the fractal dimension of rough surfaces," Tribol. Lett. 54, 99 (2014).

${ }^{22}$ B. Hubbard, M. Siegert, and D. McCarroll, "Spectral roughness of glaciated bedrock geomorphic surfaces: Implications for glacier sliding," J. Geophys. Res.: Solid Earth 105, 21295, https://doi.org/10.1029/2000jb900162 (2000).

${ }^{23}$ B. Lorenz and B. N. J. Persson, "Leak rate of seals: Effective-medium theory and comparison with experiment," Eur. Phys. J. E 31, 159 (2010).

${ }^{24}$ W. B. Dapp, A. Lücke, B. N. J. Persson, and M. H. Müser, "Self-affine elastic contacts: Percolation and leakage," Phys. Rev. Lett. 108, 244301 (2012).

${ }^{25}$ M. H. Müser, W. B. Dapp, R. Bugnicourt, P. Sainsot, N. Lesaffre, T. A. Lubrecht, B. N. J. Persson, K. Harris, A. Bennett, K. Schulze, S. Rohde, P. Ifju, W. G. Sawyer, T. Angelini, H. A. Esfahani, M. Kadkhodaei, S. Akbarzadeh, J.-J. Wu, G. Vorlaufer, A. Vernes, S. Solhjoo, A. I. Vakis, R. L. Jackson, Y. Xu, J. Streator, A. Rostami, D. Dini, S. Medina, G. Carbone, F. Bottiglione, L. Afferrante, J. Monti, L. Pastewka, M. O. Robbins, and J. A. Greenwood, "Meeting the contact-mechanics challenge," Tribol. Lett. 65, 118 (2017). 